\title{
Lateral magnetically modulated multilayers by combining ion implantation and lithography
}

Enric Menéndez*, Hiwa Modarresi, Claire Petermann, Josep Nogués*, Neus Domingo, Haoliang Liu, Brian J. Kirby, Brian Maranville, Julie A. Borchers, Amir Syed Mohd, Zahir Salhi, Earl Babcock, Stefan Mattauch, Liesbet Lagae, Chris Van Haesendonck, André Vantomme, and Kristiaan Temst*

Dr. E. Menéndez

KU Leuven, Instituut voor Kern- en Stralingsfysica, Celestijnenlaan 200 D, 3001 Leuven, Belgium

E-mail: enricmenendez@gmail.com

Prof. J. Nogués

Catalan Institute of Nanoscience and Nanotechnology (ICN2), CSIC and The Barcelona Institute of Science and Technology, Campus UAB, Bellaterra, 08193 Barcelona, Spain

ICREA-Institució Catalana de Recerca i Estudis Avançats, Barcelona, Spain

E-mail: josep.nogues@uab.cat

Prof. K. Temst

KU Leuven, Instituut voor Kern- en Stralingsfysica, Celestijnenlaan 200 D, 3001 Leuven, Belgium E-mail: kristiaan.temst@fys.kuleuven.be

H. Modarresi, C. Petermann, Prof. A. Vantomme

KU Leuven, Instituut voor Kern- en Stralingsfysica, Celestijnenlaan 200 D, 3001 Leuven, Belgium

Dr. N. Domingo

Catalan Institute of Nanoscience and Nanotechnology (ICN2), CSIC and The Barcelona Institute of Science and Technology, Campus UAB, Bellaterra, 08193 Barcelona, Spain

Dr. H. Liu, Prof. C. Van Haesendonck

KU Leuven, Laboratorium voor Vaste-Stoffysica en Magnetisme, Celestijnenlaan 200 D, 3001 Leuven, Belgium

Dr. B. J. Kirby, Dr. B. Maranville, Dr. J. A. Borchers

NIST Center for Neutron Research, National Institute of Standards and Technology, Gaithersburg, Maryland 20899, USA

Dr. A. S. Mohd, Dr. Z. Salhi, Dr. E. Babcock, Dr. S. Mattauch

Jülich Centre for Neutron Science (JCNS), Forschungszentrum Jülich GmbH, Außenstelle am FRM-II c/o TU München, Lichtenbergstraße 1, D-85747 Garching b. München, Germany

Prof. L. Lagae

KU Leuven, Laboratorium voor Vaste-Stoffysica en Magnetisme, Celestijnenlaan 200 D, 3001 Leuven, Belgium

IMEC, Kapeldreef 75, 3001 Leuven, Belgium

Keywords: spintronics, planar technology, ion implantation, lithography, magnetoresistance

"This is the peer reviewed version of the following article: E. Menéndez, H. Modarresi, C. Petermann, J. Nogués, N. Domingo, H. Liu, B. J. Kirby, A. S. Mohd, Z. Salhi, E. Babcock, S. Mattauch, C. Van Haesendonck, A. Vantomme, K. Temst. Lateral Magnetically Modulated Multilayers by Combining Ion Implantation and Lithography. Small 2017, 13, 1603465. Which has been published in final form at http://dx.doi.org/10.1002/smll.201603465.

This article may be used for non-commercial purposes in accordance with Wiley Terms and Conditions for Use of Self-Archived Versions." 


\section{Abstract}

The combination of lithography and ion implantation is demonstrated to be a suitable method to prepare lateral multilayers. A laterally, compositionally and magnetically, modulated microscale pattern consisting of alternating $\mathrm{Co}(1.6 \mu \mathrm{m}$-wide $)$ and $\mathrm{Co}-\mathrm{CoO}(2.4 \mu \mathrm{m}$-wide) lines has been obtained by oxygen ion implantation into a lithographically-masked Au-sandwiched Co thin film. Magnetoresistance along the lines (i.e., current and applied magnetic field are parallel to the lines) reveals an effective positive giant magnetoresistance (GMR) behavior at room temperature. Conversely, anisotropic magnetoresistance and GMR contributions are distinguished at low temperature (i.e., $10 \mathrm{~K}$ ) since the $\mathrm{O}$-implanted areas become exchange-coupled. This planar GMR is ascribed to the high density of $180^{\circ}$ domain walls arising from both the reversal of the non-implanted Co areas (intrinsic $180^{\circ}$ cross-tie domain walls) and the spatial modulation of coercivity in a springmagnet-type configuration, which results in $180^{\circ}$ Néel extrinsic domain walls at the $\mathrm{Co} / \mathrm{Co}-\mathrm{CoO}$ interfaces. The versatility, in terms of pattern size, morphology and composition adjustment, of this method offers a unique route to fabricate planar systems for spintronic research and applications.

\section{Introduction}

Nowadays, spintronic research and devices are mainly based on vertical rather than planar systems, such as multilayered magnetic tunnel junctions. ${ }^{[1,2]}$ That is, sandwich geometries (vertical multilayers) prevail over in-plane modulated patterns (lateral multilayers) since planar technology (e.g., planar giant magnetoresistance (GMR) readers) ${ }^{[3]}$ still remains challenging from a fabrication point of view. The preparation of lateral multilayers can be achieved, among others, by shadow evaporation techniques which combine lithography and multiple depositions, ${ }^{[4]}$ lithography and a combination of hard and soft lift-off methods, ${ }^{[5]}$ multilevel lithography and ion milling to introduce periodic thickness modulations which may result in magnetic lateral multilayers, ${ }^{[6-8]}$ self-organization through chemical instabilities $^{[9-11]}$ or ion beam assisted deposition. ${ }^{[12,13]}$ Although these methods ensure in-plane compositional and/or magnetic modulations, many of them suffer from different drawbacks like 
several intermediate fabrication steps, pattern size control, limited morphology design or non-tunable interfaces. Therefore, routes which can lead to lateral multilayers with sharp interfaces and versatility in pattern design in a minimized number of preparation stages are of great interest, not only from a technological point of view but also from a scientific side. Planar technology offers advantages when compared to standard vertical technology (e.g., devices are finished at the wafer level ${ }^{[3]}$ and more flexibility to perform spatially-resolved characterization due to its lateral geometry ${ }^{[14]}$ In this context, ion irradiation/implantation in combination with lithography has become a suitable method to obtain lateral multilayers ${ }^{[15-18]}$ and magnetically pattern systems. ${ }^{[15,19-22]}$

Ion implantation is a suitable technique to controllably modify the near-surface of materials due to the limited range of penetration of ions, ${ }^{[23]}$ turning out to be particularly convenient to tune the structure and composition of thin films. Light-ion irradiation (mainly of noble gases) of ferromagnetic (FM) thin films and multilayers has been used for the modification of magnetic properties, such as magnetic anisotropy, saturation magnetization, exchange coupling or exchange bias. ${ }^{[24]}$ Furthermore, irradiation using heavy ions and ion implantation have also been utilized with the aim to induce increased collisional damage and/or to create composition adjustments. ${ }^{[25]}$ Recently, oxygen ion implantation has been confirmed as an advantageous route to form antiferromagnetic (AFM) oxides embedded in FM thin films (e.g., Co), giving rise to exchange bias. ${ }^{[26-31]}$ In parallel, the wide variety of lithography techniques yields a high flexibility in pattern design and, thus, in shadow mask fabrication for ion implantation.

We have combined O ion implantation with UV lithography in order to prepare $\mathrm{Co} / \mathrm{Co}-\mathrm{CoO}$ microscale lines with laterally modulated magnetic properties, which give rise to planar GMR effects.

\section{Results and discussion}




\subsection{Preparation of laterally modulated $\mathrm{Co} / \mathrm{Co}-\mathrm{CoO}$ micropatterns by combining $\mathrm{O}$ ion implantation}

and lithography

An Au-sandwiched Co thin film (i.e., $20 \mathrm{~nm} \mathrm{Au/} 30 \mathrm{~nm} \mathrm{Co} / 10 \mathrm{~nm} \mathrm{Au}$ ) was grown by molecular beam epitaxy onto a thermally-oxidized Si(100) wafer. The top 20 nm-thick Au layer (i.e., capping layer) prevents Co from natural oxidation, while the bottom $10 \mathrm{~nm}$-thick Au layer (i.e., buffer layer) is introduced to minimize the atomic intermixing between $\mathrm{Co}$ and $\mathrm{SiO}_{2}$ upon implantation. Both Au layers are intended to avoid any other source of $\mathrm{CoO}$ formation than that of the $\mathrm{O}$ ion implantation. The Co layer is polycrystalline and consists of a mixture of face-centered cubic $\mathrm{Co}$, hexagonal close-packed Co and stacking faults. ${ }^{[29]}$

Microscale patterns consisting of $1.6 \mu \mathrm{m}$-wide lines with a period of $4 \mu \mathrm{m}$ were prepared by UV lithography. Since the lithographed resist is intended to be used as a shadow mask for ion implantation, an additional Au layer of $10 \mathrm{~nm}$ was deposited to further ensure the full stopping of the impinging ions by the resist during implantation (Figure 1a). The sample was then implanted with $45 \mathrm{keV} O$ ions to a fluence of $2 \times 10^{17}$ ions $/ \mathrm{cm}^{2}$ and, afterwards, the remaining resist stripped away.

The $\mathrm{O}$ ion implantation into the resist-free areas leads to a fairly uniform $\mathrm{O}$ depth profile across the Co layer (Figure 1b), whereas, as confirmed by $\operatorname{TRIM}^{[32]}$ simulations (not shown), the shadow mask (i.e., $10 \mathrm{~nm}$ Au-resist) guarantees the full stopping of the incoming $\mathrm{O}$ ions (Figure 1c). In this fashion, the sample is selectively implanted, resulting in a periodic formation of $\mathrm{CoO} .{ }^{[29]}$ Hence, the combination of $\mathrm{O}$ ion implantation and lithography yields a laterally modulated pattern consisting of alternating Co and Co-CoO microscale lines sandwiched between Au layers (Figure 1c).

\subsection{Room temperature magnetic and transport properties}

Figures $2 a$ and $2 b$ show the longitudinal and transversal magneto-optic Kerr effect (MOKE) measurements, respectively, along and across the lines. The longitudinal MOKE measurement along the lines reveals a double-step-like hysteresis loop. This is the result of the interplay between the dissimilar coercivities of the non- and implanted areas (being the implanted lines magnetically harder) and dipolar interactions, which tend to stabilize the antiparallel alignment of adjacent lines once the 
magnetization of the soft areas (i.e., non-implanted lines) is switched. The implantation leads to an increase in the number of defects in Co, such as stacking faults, that can act as pinning centers for magnetization reversal, resulting in an enhanced coercivity. ${ }^{[29,33]}$ Upon implantation, oxygen primarily accumulates at the grain boundaries forming $\mathrm{CoO}$, reducing the grain size and thus isolating the Co grains. This might also yield decreased exchange interactions among Co grains leading to an increased coercivity. Furthermore, the implantation-induced increase of coercivity can also be partially ascribed to exchange interactions between the $\mathrm{Co}$ grains and the $\mathrm{CoO}$ at the grain boundaries. ${ }^{[29,34]}$ Conversely, the longitudinal MOKE measurement across the lines shows a single-step hysteresis loop with slightly decreased coercivity and remanence, evidencing traces of shape anisotropy. In this case, once the magnetization of the pure Co lines is switched, the dipolar fields favor the reversal of the implanted areas, softening the jumps in magnetization and thus yielding a more gradual magnetization reversal. As can be seen in Figure $2 \mathrm{~b}$, the transversal MOKE measurement along the lines reveals traces of perpendicular magnetization upon reversal, indicating that coherent rotation is an active magnetization reversal mechanism. The slightly broadened transversal signal for the measurement across the lines confirms the mild shape anisotropy already envisaged by longitudinal MOKE, since coherent rotation typically governs the magnetization reversal along hard axes. ${ }^{[35]}$

Magnetoresistance, $\Delta R / R$, with the current and external magnetic field applied along the patterned lines exhibits an overall positive effect (Figure 2c) for both descending and ascending branches. Lorentz magnetoresistance, originating from the deflection of the current lines inside magnetic domains, and the magnetoresistance effect linked to the twisting of the current lines at the domain walls (i.e., Hall effect contribution) can be neglected due to the polycrystalline nature of the sample. ${ }^{[36]}$ Thus, the observed $\Delta R / R$ might at first glance be ascribed to anisotropic magnetoresistance (AMR), which arises from spin-orbit coupling. However, in this configuration, AMR contributions should result in a decrease of the resistance regardless of the magnetization reversal mechanism since the change in AMR is proportional to $\cos ^{2} \theta$, where $\theta$ is the angle between the current and the magnetization. ${ }^{[37]}$ Hence, this implies that, in the $\mathrm{Co}-\mathrm{Co} / \mathrm{CoO}$ lateral multilayers, AMR is overplayed by another magnetoresistance 
effect. The maxima of magnetoresistance appear between -7 and $7 \mathrm{mT}$ of applied magnetic field. As can be seen in the longitudinal MOKE measurement along the lines (Figure 2a), the soft magnetic areas (i.e., non-implanted) reverse their magnetization in this field range, whereas the magnetization of the implanted lines remains essentially unaltered. To further unravel the magnetic domain configurations upon magnetization reversal, magnetic force microscopy (MFM) images were taken at remanence (after saturating the sample along the lines in a field of $-100 \mathrm{mT}$ ) and at increasingly positive applied magnetic fields along the lines (Figure 3). Figure 3a, taken at remanence, reveals a multidomain magnetic structure of the pure Co lines in agreement with the longitudinal MOKE characterization which shows that the remanence is lower than the saturation magnetization. Upon increasing the applied magnetic while keeping the main orientation of the magnetization of the non-implanted areas unchanged (Figures $3 \mathrm{~b}-3 \mathrm{~d}$ ), the multidomain structure reinforces and $180^{\circ}$ cross-tie domain walls (domain walls which contain both Néel- and Bloch-like counterparts) ${ }^{[38-41]}$ can be locally observed within the O-free Co lines. As can be seen in Figures $3 \mathrm{~d}$ and $3 \mathrm{e}$, the switch of the main orientation of the magnetization takes place in an applied magnetic field interval of less than $0.5 \mathrm{mT}$ in fair concordance with the narrow switching field range of the non-implanted lines (Figure 2a). Differences in applied field dependences among MOKE, magnetoresistance and MFM characterization are mainly ascribed to the local probing character of these techniques and the fact that the measurements were independently carried out. Upon switching of the soft magnetic parts, Néel-like domain walls ${ }^{[42]}$ at the $\mathrm{Co} / \mathrm{Co}-\mathrm{CoO}$ interfaces (see the magnetization configuration scheme of Figure $3 \mathrm{e}$ ) are envisaged until the implanted areas fully reverse. This indicates that, during and after magnetization reversal of the pristine Co areas, a local antiparallel alignment of the magnetization (via $180^{\circ}$ cross-tie- and interface $180^{\circ}$ Néel-like domain walls, respectively) is present. This interface $180^{\circ}$ Néel-like domain walls have been already reported, among others, in Co-based ${ }^{[43]}$ and Fe-based ${ }^{[44-46]}$ systems. Therefore, taking into account this local antiparallel configuration, the observed positive magnetoresistance (Figure 2c) can be linked to a domain wall resistance effect ruled by giant magnetoresistance mechanisms. ${ }^{[43,47]}$ Since domain walls in single Co thin films do not significantly scatter conduction electrons (i.e., GMR 
effects are not dominant sources of magnetoresistance) ${ }^{[48]}$ this confirms the high density of domain walls achieved in this system, which is ascribed to both intrinsic (e.g., magnetocrystalline anisotropy of Co) and extrinsic (e.g., pattern design, Co thickness, shape anisotropy...) magnetic properties. Both descending and ascending branches exhibit a magnetoresistance maximum, suggesting that the formation of these $180^{\circ}$ domain walls is, to some extent, reversible. The maximum variation with respect to the resistance at saturation is around $\Delta R / R(\%)=0.03$, whose order of magnitude is consistent with the domain wall resistance of other Co-based systems. ${ }^{[43]}$ Nevertheless, although the effects are unambiguous, the $\Delta R / R$ values are somewhat limited by the shunting effects of the $A u$ capping and buffer layers and the difference in resistance between the Co and Co-CoO lines (which tends to favor the flow of current through the Co layer).

\subsection{Low temperature magnetic and transport properties}

For the low temperature characterization (i.e., magnetometry, magnetoresistance and polarized neutron reflectometry), the sample was cooled from room temperature down to $10 \mathrm{~K}$ in a magnetic field of $800 \mathrm{mT}$ applied along the lines. Figure 4a shows the superconducting quantum interference device (SQUID) measurements of consecutively measured hysteresis loops (i.e., untrained and trained) along the lines. These double-step cycles are the result of a combination of soft and hard magnetic contributions, which originate from the pure Co and the O-implanted lines, respectively. In contrast to room temperature, the coercivity of the O-implanted Co lines is significantly enhanced. Together with the pronounced exchange bias shift ${ }^{[49,50]}$ (around $-92.7 \mathrm{mT}$ ), this confirms that the $\mathrm{Co}$ and the $\mathrm{CoO}$ in the implanted areas are exchange-coupled, in agreement with previously reported results. ${ }^{[29,31]}$ The trained loop shows a decreased exchange bias shift of $-62.8 \mathrm{mT}$ in concordance with the strong training effects that occur in Co-CoO systems. ${ }^{[31]}$ The reversal of the implanted areas takes place within a relatively narrow switching field range, corroborating the homogeneity of the $\mathrm{O}$ profile simulated by TRIM. $^{[32]}$ Remarkably, this lateral configuration of exchange-biased (Co-CoO) and non-biased (Co) alternating regions gives rise to a well-defined spatially modulated coercivity. 
As can be seen in Figure $4 \mathrm{~b}$, parallel magnetoresistance (i.e., current and $\mathrm{H}_{\text {applied }}$ along the lines) shows traces of positive and negative contributions for both untrained and trained branches. Whereas the maxima appear in the field range where the non-implanted areas reverse, the minima emerge in the field region corresponding to the reversal of O-implanted areas. With consecutively measured loops, the positive contributions remain virtually unaltered, while the minima take place at decreased applied magnetic fields, evidencing training ${ }^{[49,50]}$ and, thus, indicating that the negative contributions originate from the implanted areas which are exchange-coupled. In the second measurement (i.e., trained), the depth of the minimum is enhanced because of the additional spin rotation resulting from the training effect. $^{[31]}$

To further shed light on this magnetoresistance behavior, the magnetization reversal mechanisms have been investigated by polarized neutron reflectometry (PNR) with off-specular analysis. The PNR magnetic field scans acquired by integrating the full signal (i.e., specular and off-specular) are presented in Figure 5. In the field region where the implanted areas inverse their magnetization, no spin flip signal (ud and du) above the background level can be observed, indicating that the magnetization reversal is mainly ruled by domain wall nucleation and motion in agreement with the results of homogeneously implanted Co thin films. ${ }^{[31]}$ Conversely, a large spin flip signal is detected in the field range where the pristine Co lines reverse. The corresponding non-spin flip signals (uu and dd) cross roughly halfway between the minimum and maximum values, suggesting the existence of another source of spin flip signal on top of that arising from coherent rotation. The disentanglement of the specular and off-specular signals in the magnetic field scans (not shown) indicates that most of the spin flip signal arises from diffuse (i.e., off-specular) magnetic scattering (i.e., in-plane magnetic correlations). This is consistent with a magnetization reversal of the Co lines governed by domain wall nucleation and motion with coherent rotation traces, in agreement with the room temperature transversal MOKE characterization.

Figure 6 shows the $\alpha_{i}-\alpha_{f}$ maps of the reflected intensity corresponding to uu (a), dd (b), ud (c) and ud (d) neutrons, recorded in an applied magnetic field of $-0.6 \mathrm{mT}$. This value was chosen with a two-fold 
aim. That is, to avoid depolarization of the neutron beam (see Experimental Section) and to investigate the remnant state of the O-free Co areas where, as can be seen in Figure 4, the magnetization of the Co areas has partially reversed $\left(M_{R}<M_{S}\right.$, with $M_{R}$ and $M_{S}$ the magnetization at remanence and at saturation, respectively), allowing the study of the magnetization reversal of the O-free Co areas. The off-specular Bragg peaks of the non spin flip signals (panels (a) and (b)) confirm the high lateral topographic correlation of the sample, while the off-specular fringes of the spin flip signal maps reveal in-plane magnetic correlations, in concordance with the large spin flip signal observed in the magnetic field scans of Figure 5. This is in agreement with previously reported off-specular PNR results on Co thin films where lateral networks of Néel domain walls are also responsible for the off-specular signal. ${ }^{[51]}$ The asymmetry (i.e., spin dependence) in the diffuse spin flip scattering, where ud does not equal du, is ascribed to a birefringence phenomenon. ${ }^{[52-55]}$ In this context, Zeeman splitting is ruled out as a source of off-specular spin-flip signal since it is in moderate and strong external magnetic fields. ${ }^{[56,57]}$ Conversely, in the field range where the magnetization of the implanted areas reverse and further (i.e., saturation), no traces of off-specular signal are observed, indicating no in-plane magnetic correlations, suggesting that domain walls with partial or antiparallel alignment are much scarcer or negligible with respect to the pure Co areas. This is in agreement with the fact that AMR prevails over GMR as the dominant source of magnetoresistance in the implanted areas. Taking this into account, the small negative contribution to the magnetoresistance of the untrained descending branch (Figure 4b) can be explained by weak AMR effects originating from minor perpendicular contributions of the magnetization during domain wall nucleation and motion. This negative resistance contribution becomes more pronounced with consecutive reversals (training), suggesting that coherent rotation reinforces since the corresponding perpendicular components of the magnetization are significantly larger (being largest at the coercive fields) than those of domain wall nucleation and motion processes. Even though this evolution is consistent with the reversal mechanism asymmetry in $\mathrm{Co} / \mathrm{CoO}$ bilayers and O-implanted systems with low $\mathrm{O}$ contents, these negative contributions are weak $(0.11 \%$ for the trained descending branch), in concordance with the PNR results which indicate that the reversals of 
implanted areas are mainly ruled by domain wall nucleation and motion (Figure 5 ). In fact, reversals fully taking place via coherent rotation would lead to much more pronounced changes in resistance, as it happens in $\mathrm{Co} / \mathrm{CoO}$ bilayers where magnetoresistance differences up to $80 \%$ are observed. ${ }^{[58]}$ On the contrary, in the field range where the Co lines inverse their magnetization, the resistance, rather than showing a decrease, exhibits an upturn, which screens any AMR effects arising from domain wall nucleation and motion and coherent rotation traces. As can be seen in Figure $4 b$, this increase is already significant where most of the magnetization has not yet reversed (remanence is above $92 \%$ for the first four reversals, Figure 4a), in consonance with the existence of intrinsic $180^{\circ}$ cross-tie domain walls within the Co areas. As it happens at room temperature, these incipient positive magnetoresistance effects can be linked to domain wall resistance contributions through a giant magnetoresistance mechanism. ${ }^{[43,47]}$ Nonetheless, the maxima of spin flip signal occur when the magnetization of the Co areas is half reversed (i.e., at the coercive fields, Figure 5), whereas the magnetoresistance reaches its maximum when the Co areas are fully reversed (i.e., at around $-16 \mathrm{mT}$ for the descending branches). This implies an additional source of positive magnetoresistance which can be linked to the $180^{\circ}$ Néel-like domain walls arising from the antiparallel alignment of adjacent lines upon reversal of the soft areas (Figure 4c). Namely, the anti-parallel coupling results in $180^{\circ}$ Néellike domain walls at the $\mathrm{Co} / \mathrm{Co}-\mathrm{CoO}$ interfaces and it consequently yields an additional increase of the resistance due to a giant magnetoresistance mechanism. In contrast to the intrinsic Néel domain walls arising from the magnetization reversal of Co lines, these interfacial domain walls are of extrinsic origin (i.e., artificial) since they originate from magnetic periodicity arising from the dissimilar coercivity of contiguous lines. Actually, this also applies to the room temperature magnetoresistance, whose interpretation is less straightforward since the magnetic contributions of non- and implanted lines are more entangled due to the smaller difference in coercive fields. The positive contribution reaches values with respect to the resistance at saturation up to around $\Delta R / R(\%)=0.13$, close to four times larger than at room temperature, evidencing the partial disentanglement of the AMR contribution ascribed to exchange-coupled areas. These magnetoresistance maxima appear in both descending and 
ascending branches and do not suffer from training, indicating a high reversibility in the formation of these $180^{\circ}$ domain walls.

\section{Conclusion}

Ion implantation through lithographed masks is shown to be an advantageous method to prepare laterally, compositionally and magnetically, modulated samples with tunable giant magnetoresistance and potential uses for spintronic research and applications. A lateral microscale pattern consisting of alternating Co (1.6 $\mu \mathrm{m}$-wide) and Co-CoO (2.4 $\mu \mathrm{m}$-wide) lines has been prepared by oxygen ion implantation into a pre-lithographed Au-sandwiched Co thin film. The lithography is used to obtain a shadow mask for ion implantation. By locally implanting $\mathrm{O}$ into a Co thin film, the magnetic properties can be periodically altered in the plane of the sample, e.g., in our case, a spatially modulated coercivity is achieved. Consequently, domain structures can be imposed by the application of well-defined magnetic fields. This magnetic periodicity leads to an overall positive giant magnetoresistance behavior at room temperature, while anisotropic and giant magnetoresistance contributions are distinguished at low temperature since the O-implanted areas become exchange-coupled. This planar giant magnetoresistance is ascribed to the high density of $180^{\circ}$ domain walls arising from both the reversal of the non-implanted Co areas (intrinsic $180^{\circ}$ cross-tie-like domain walls) and the spatial modulation of coercivity (in a spring-magnet-type configuration), which results in $180^{\circ}$ Néel-like domain walls at the $\mathrm{Co} / \mathrm{Co}-\mathrm{CoO}$ interfaces. This novel approach may constitute an important asset for planar spintronics and other magnetic lateral multilayer applications.

\section{Experimental Section}

A $1.5 \times 1.5 \mathrm{~cm}^{2}$ Au-sandwiched polycrystalline $30 \mathrm{~nm}$-thick Co thin film (i.e., $20 \mathrm{~nm} \mathrm{Au/} 30 \mathrm{~nm} \mathrm{Co/} 10$ $\mathrm{nm} \mathrm{Au}$ ) was grown by molecular beam epitaxy at room temperature on thermally-oxidized Si (100) 
substrates $\left(450 \mathrm{~nm}\right.$-thick $\left.\mathrm{SiO}_{2}\right)$. All layers were grown at a pressure of around $3 \times 10^{-10} \mathrm{mbar}$. The Co layer consists of a mixture of face-centered cubic $\mathrm{Co}$, hexagonal close-packed $\mathrm{Co}$ and stacking faults. ${ }^{[29]}$ UV lithography was used to design microscale lines on top of the sample. In order to achieve a fine undercutting of the resist profile, a non-photosensitive layer (Shipley, MICROPOSIT ${ }^{\bullet}$ LOL2000) was first spin-coated onto the sample for $50 \mathrm{~s}$ at $4000 \mathrm{rpm}$, resulting in a thickness of around $0.16 \mu \mathrm{m}$. Then, the sample was soft-baked for five minutes at $115^{\circ} \mathrm{C}$. Subsequently, a photosensitive layer (Shipley, MICROPOSIT ${ }^{\circ}$ S1813) was spin-coated on top of the first resist for $50 \mathrm{~s}$ at $5000 \mathrm{rpm}$, yielding a total thickness of approximately $1.2 \mu \mathrm{m}$. The resist system was then soft-baked at $115^{\circ} \mathrm{C}$ for $1 \mathrm{~min}$. The sample was afterwards subjected to illumination through a pre-designed mask consisting of $2 \mu \mathrm{m}$-wide lines with a period of $4 \mu \mathrm{m}$ with UV light from a short-arc mercury vapor lamp (Osram, HBO 200W/4). The time of exposure was about $10 \mathrm{~s}$. The exposed sample was then submerged into a developer solution (Shipley, MICROPOSIT ${ }^{\bullet}$ MF319), which dissolves the illuminated parts of the resist layer (positive resist). Since the development rate of the underlying LOL2000 layer is higher than that of the S1813 resist and the non-photosensitive LOL2000 layer dissolves in a controllable way, well-defined and reproducible undercuts of the top resist layer can be achieved. The development time was $30 \mathrm{~s}$. While developing, the exposed resist is removed and dissolution of the photoresist slows down, but the developer continues to dissolve the LOL2000 layer in the open areas and under the resist edge, leading to well-defined structures. The dimensions of the pattern were checked by optical microscopy, indicating the formation of $1.6 \mu \mathrm{m}$-wide lines with a period of $4 \mu \mathrm{m}$ (the lines constitute the $40 \%$ of the sample surface and the non-covered areas the $60 \%$ ).

Subsequently, with the aim to further increase the thickness of the resist layer, $10 \mathrm{~nm}$ of Au were additionally sputtered on top. The lithographed sample was then implanted using $45 \mathrm{keV} O$ ions to a fluence of $2 \times 10^{17}$ ions $/ \mathrm{cm}^{2}$.

The magnetic properties were investigated, at room temperature, by means of magneto-optical Kerr effect (MOKE) measurements and, at low temperature, by superconducting quantum interference device (SQUID) magnetometry. With the aim to probe several lines, the laser of the MOKE setup was 
defocused up to $50 \mu \mathrm{m}$ in diameter. Magnetic force microscopy (MFM) with in-situ applied magnetic field was used to further unravel the magnetization reversal at room temperature.

High-resolution four-terminal magnetoresistance measurements were performed both at room temperature and at $10 \mathrm{~K}$ (after field cooling from room temperature in a magnetic field of $800 \mathrm{mT}$ applied parallel to the lines) in a helium flow cryostat by integrating the sample into an Adler-Jackson bridge. The $\mathrm{AC}$ measuring current for the lock-in detection has a frequency of $27.7 \mathrm{~Hz}$ and a root-meansquare amplitude of 3.5 A. The current direction was established along the lines.

Polarized neutron reflectometry (PNR) with off-specular analysis was carried out at the MARIA instrument, operated by JCNS at Heinz Maier-Leibnitz Zentrum (MLZ), Garching, Germany. A neutron wavelength of $6 \AA$ was used. Specifically, PNR magnetic field scans were carried out to unravel the magnetization reversal mechanisms along the lines at low temperature after parallel field cooling. From the specular polarized reflectivity pattern recorded in the saturated magnetization state, the angle (i.e., the incidence angle of neutrons) showing a good tradeoff between intensity and splitting ratio was selected to perform these magnetic field scans. That is, at a certain fixed angle (i.e., $0.96^{\circ}$ $\left.\left(0.035 \AA^{-1}\right)\right)$, the non-spin flip (NSF) and the spin flip (SF) signals are recorded as a function of the applied magnetic field. ${ }^{[59,60]}$ Upon reflection, the neutron polarization is analyzed, resulting in four different measured reflectivities: two NSF signals, uu and dd, and two SF signals, ud and du. The first index denotes the polarization prior to reflection and the second index the polarization after reflection. In order to maintain the polarization of the neutrons throughout the reflectometer, guide fields are mounted at dedicated positions. Since neutrons depolarize due to stray fields when a positive field is applied, the measurements can only be performed at negative fields. Hence, in order to assess the ascending branches (which usually lie at positive fields after positive field cooling), the sample was cooled in a negative field, implying that the aforementioned ascending branches will then reside at negative fields. The ascending and descending branches were in fact measured after separate field cooling processes, which explains why the magnetic field scans always have a negative magnetic field scale. Moreover, two dimensional reflectivity measurements $\left([]_{i}-[]_{f}\right.$ maps containing the specular and 
off-specular signals) were performed at dedicated applied magnetic fields (Figure 6). The polarized and analyzed data of Figure 6 are corrected according to reference [61] after taking into account that MARIA is equipped with a polarizing supermirror (double bounce) as polarizer and an in-situ SEOP ${ }^{3} \mathrm{He}-$ filter as analyzer ${ }^{[62]}$ The latter is combined with a transverse RF-field performing an adiabatic fast passage spin reversal of the ${ }^{3} \mathrm{He}$ and used instead of a neutron flipper. In this way, the analyzer has in both directions the same efficiency. On the side of the polarizer, the neutrons are flipped with an RFflipper which is working close to 100\%. Therefore the data shown in Figure 6 are corrected for the polarization level of $98 \%$, removing the leakage in the spin flip channels.

\section{Acknowledgements}

This work was financed by the Research Foundation-Flanders (FWO), the Concerted Research Action GOA/14/007, the 2014-SGR-1015 project of the Generalitat de Catalunya, and the European Commission under the $7^{\text {th }}$ Framework Programme through the "Research Infrastructure" action of the "Capacities" Programme, NMI3-II Grant No. 283883. This work is based upon experiments (proposals numbers 5928 and 9384) performed at the MARIA instrument operated by JCNS at Heinz MaierLeibnitz Zentrum (MLZ), Garching, Germany. The authors would like to thank IMEC for access to its lithography facilities as well as J. Moonens and J. Loo for technical support. ICN2 acknowledges support from the Severo Ochoa Program (MINECO, Grant SEV-2013-0295).

[1] I. Zutic, J. Fabian, S. Das Sarma, Rev. Mod. Phys. 2004, 76, 323.

[2] S. D. Bader, S. S. P. Parkin, Annu. Rev. Condens. Matter Phys. 2010, 1, 71.

[3] E. D. Tober, R. F. Marks, D. D. Chambliss, K. P. Roche, M. F. Toney, A. J. Kellock, R. F. C. Farrow, Appl. Phys. Lett. 2000, 77, 2728.

[4] T. Kimura, T. Sato, Y. Otani, Phys. Rev. Lett. 2008, 100, 066602.

[5] A. O. Adeyeye, M. K. Husain, and V. Ng, J. Magn. Magn. Mater. 2002, 248, 355.

[6] A. Hierro-Rodriguez, G. Rodríguez-Rodríguez, J. M. Teixeira, G. N. Kakazei, J. B. Sousa, M.

Vélez, J. I. Martín, L. M. Alvarez-Prado, J. M. Alameda, J. Phys. D: Appl. Phys. 2013, 46, 345001.

[7] A. Hierro-Rodriguez, M. Vélez, R. Morales, N. Soriano, G. Rodríguez-Rodríguez, L. M. ÁlvarezPrado, J. I. Martín, J. M. Alameda, Phys. Rev. B 2013, 88, 174411. 
[8] A. Hierro-Rodriguez, R. Cid, M. Vélez, G. Rodriguez-Rodriguez, J. I. Martín, L. M. ÁlvarezPrado, J. M. Alameda, Phys. Rev. Lett. 2012, 109, 117202.

[9] E. D. Tober, R. F. C. Farrow, R. F. Marks, G. Witte, K. Kalki, D. D. Chambliss, Phys. Rev. Lett. 1998, 81, 1897.

[10] B. Borca, O. Fruchart, C. Meyer, J. Appl. Phys. 2006, 99, 080514.

[11] I. Golvano-Escobal, B. Özkale, S. Suriñach, M. D. Baró, T. Dobrovolska, I. Krastev, S. Pané, J. Sort, E. Pellicer, J. Mater. Chem. C 2014, 2, 8259.

[12] M. Krause, A. Muecklich, T. W. H. Oates, M. Zschornak, S. Wintz, J. L. Endrino, C. Baehtz, A. Shalimov, S. Gemming, G. Abrasonis, Appl. Phys. Lett. 2012, 101, 053112.

[13] G. Abrasonis, K. Morawetz, Phys. Rev. B 2012, 86, 085452.

[14] J. P. Nozières, E. Saporito, B. Viala, J. Magn. Magn. Mater. 2002, 242-245, 341.

[15] C. Chappert, H. Bernas, J. Ferre, V. Kottler, J.P. Jamet, Y. Chen, E. Cambril, T. Devolder, F. Rousseaux, V. Mathet, H. Launois, Science 1998, 280, 1919.

[16] N. Martin, I. Mönch, R. Schäfer, J. Fassbender, L. Schultz, J. McCord, Phys. Rev. B 2011, 83, 174423

[17] B. Obry, T. Meyer, P. Pirro, T. Brächer, B. Lägel, J. Osten, T. Strache, J. Fassbender, B. Hillebrands, Appl. Phys. Lett. 2011, 102, 174423.

[18] R. Bali, S. Wintz, F. Meutzner, R. Hübner, R. Boucher, A. A. Ünal, S. Valencia, A. Neudert, K. Potzger, J. Bauch, F. Kronast, S. Facsko, J. Lindner, J. Fassbender, Nano Lett. 2014, 14, 435.

[19] E. Menéndez, A. Martinavicius, M. O. Liedke, G. Abrasonis, J. Fassbender, J. Sommerlatte, K. Nielsch, S. Suriñach, M. D. Baró, J. Nogués, J. Sort, Acta Mater. 2008, 56, 4570.

[20] E. Menéndez, J.-C. Stinville, C. Tromas, C. Templier, P. Villechaise, J.-P. Rivière, M. Drouet, A. Martinavičius, G. Abrasonis, J. Fassbender, M. D. Baró, J. Sort, J. Nogués, Appl. Phys. Lett. 2010, 96, 242509.

[21] E. Menéndez, J. Sort, M. O. Liedke, J. Fassbender, T. Gemming, A. Weber, L. J. Heyderman, S. Suriñach, K. V. Rao, S. C. Deevi, M. D. Baró, J. Nogués, Small 2009, 5, 229-234. 
[22] A. Varea, E. Menéndez, J. Montserrat, E. Lora-Tamayo, A. Weber, L. J. Heyderman, S. C.

Deevi, K. V. Rao, S. Suriñach, M. D. Baró, K. S. Buchanan, J. Nogués, J. Sort, J. Appl. Phys. 2011, 109, 093918.

[23] M. Nastasi, J. W. Mayer, J. K. Hirvonen, lon-solid interactions: fundamentals and applications, Cambridge University Press, Cambridge, USA 1996.

[24] J. Fassbender, D. Ravelosona, Y. Samson, J. Phys. D: Appl. Phys. 2004, 37, R179.

[25] J. Fassbender, J. McCord, J. Magn. Magn. Mater. 2008, 320, 579.

[26] J. Demeter, J. Meersschaut, F. Almeida, S. Brems, C. Van Haesendonck, A. Teichert, R. Steitz, K. Temst, A. Vantomme, Appl. Phys. Lett. 2010, 96, 132503.

[27] J. Demeter, E. Menéndez, A. Vantomme, K. Temst, J. Appl. Phys. 2011, 110, 123902.

[28] J. Demeter, E. Menéndez, A. Schrauwen, A. Teichert, R. Steitz, S. Vandezande, A. R. Wildes, W. Vandervorst, K. Temst, A. Vantomme, J. Phys. D: Appl. Phys. 2012, 45, 405004.

[29] E. Menéndez, J. Demeter, J. Van Eyken, E. Jedryka, M. Wójcik, P. Nawrocki, J. Nogués, J. F. Lopez-Barbera, A. Vantomme, K. Temst, ACS Appl. Mater. Interfaces 2013, 5, 4320.

[30] E. Menéndez, H. Modarresi, T. Dias, J. Geshev, L. M. C. Pereira, K. Temst, A. Vantomme, J. Appl. Phys. 2014, 115, 133915.

[31] E. Menéndez, T. Dias, J. Geshev, J. F. Lopez-Barbera, J. Nogués, R. Steitz, B. J. Kirby, J. A. Borchers, L. M. C. Pereira, A. Vantomme, K. Temst, Phys. Rev. B 2014, 89, 144407.

[32] http://srim.org/. The TRIM (Transport of lons in Matter) program is included in the SRIM (Stopping Range of lons in Matter) package, accessed: November, 2014.

[33] J. Sort, S. Suriñach, J. S. Muñoz, M. D. Baró, M. Wojcik, E. Jedryka, S. Nadolski, N. Sheludko, J. Nogués, Phys. Rev. B 2003, 68, 014421.

[34] C. Leighton, H. Suhl, M. J. Pechan, R. Compton, J. Nogués, I. K. Schuller, J. Appl. Phys. 2002, $92,1483$.

[35] S. Chikazumi, Physics of Magnetism, John Wiley \& Sons Inc., New York, USA 1964.

[36] L. Berger, J. Appl. Phys. 1978, 49, 2156. 
[37] K. Hong, N. Giordano, Phys. Rev. B 1995, 51, 9855.

[38] H.-N. Lin, Y. H. Chiou, B.-M. Chen, H.-P. D. Shieh, C.-R. Chang, J. Appl. Phys. 1998, 83, 4997.

[39] Y. C. Chang, C. C. Chang, W. Z. Hsieh, H. M. Lee, J. C. Wu, IEEE Trans. Magn. 2005, 41, 959.

[40] A. Kharmouche, S.-M. Chérif, A. Bourzami, A. Layadi, G. Schmerber, J. Phys. D: Appl. Phys. 2004, 37, 2583.

[41] M. Löhndorf, A. Wadas, H. A. M. van den Berg, R. Wiesendanger, Appl. Phys. Lett. 1996, 68, 3635.

[42] M. Cartier, S. Auffret, Y. Samson, P. Bayle-Guillemaud, B. Dieny, J. Magn. Magn. Mater. 2001, $223,63$.

[43] D. Buntinx, S. Brems, A. Volodin, K. Temst, C. Van Haesendonck, Phys. Rev. Lett. 2005, 94, 017204.

[44] S. N. Gordeev, J. M. L. Beaujour, G. J. Bowden, B. D. Rainford, P. A. J. de Groot, R. C. C. Ward, M. R. Wells, A. G. M. Jansen, Phys. Rev. Lett. 2001, 87, 186808.

[45] S. Vandezande, C. Van Haesendonck, K. Temst, Appl. Phys. Lett. 2009, 94, 192501.

[46] J. Swerts, K. Temst, M. J. Van Bael, C. Van Haesendonck, Y. Bruynseraede, Appl. Phys. Lett. 2003, 82, 1239.

[47] P. M. Levy, S. Zhang, Phys. Rev. Lett. 1997, 79, 5110.

[48] A. D. Kent, J. Yu, U. Rudiger, S. S. P. Parkin, J. Phys. Cond. Mat. 2001, 13, R461.

[49] J. Nogués, I. K. Schuller, J. Magn. Magn. Mater. 1999, 192, 203.

[50] J. Nogués, J. Sort, V. Langlais, V. Skumryev, S. Suriñach, J. S. Muñoz, M .D. Baró, Phys. Rep. $2005,422,65$.

[51] F. Radu, V. Leiner, K. Westerholt, H. Zabel, J. McCord, A. Vorobiev, J. Major, D. Jullien, H. Humblot, F. Tasset, J. Phys.: Condens. Matter 2005, 17, 1711.

[52] T. Saerbeck, N. Loh, D. Lott, B. P. Toperverg, A. M. Mulders, M. Ali, B. J. Hickey, A. P. J. Stampfl, F. Klose, R. L. Stamps, Phys. Rev. B 2012, 85, 014411.

[53] E. Kentzinger, U. Rücker, B. Toperverg, F. Ott, T. Brückel, Phys. Rev. B 2008, 77, 104435. 
[54] B. Toperverg, O. Nikonov, V. Lauter-Pasyuk, H. J. Lauter, Phys. B 2001, 297, 169.

[55] A. Paul, T. Krist, A. Teichert, R. Steitz, Phys. B 2011, 406, 1598.

[56] G. P. Felcher, S. Adenwalla, V. O. de Haan, A.A. van Well, Nature 1995, 377, 409.

[57] R. W. E. van de Kruijs, H. Fredrikze, M. Th. Rekveldt, A. A. van Well, Yu. V. Nikitenko, V. G. Syromyatnikov, Phys. B 2000, 283, 189.

[58] S. Brems, D. Buntinx, K. Temst, C. Van Haesendonck, F. Radu, H. Zabel, Phys. Rev. Lett. 2005, 95, 157202.

[59] G. P. Felcher, R. O. Hilleke, R. K. Crawford, J. Haumann, R. Kleb, G. Ostrowski, Rev. Sci. Instrum. 1987, 58, 609.

[60] J. Demeter, A. Teichert, K. Kiefer, D. Wallacher, H. Ryll, E. Menéndez, D. Paramanik, R. Steitz, C. Van Haesendonck, A. Vantomme, K. Temst, Rev. Sci. Instrum. 2011, 82, 033902.

[61] A. R. Wildes, Rev. Sci. Instrum. 1999, 70, 4241.

[62] E. Babcock, A. loffe, J. Phys.: Conf. Ser. 2011, 294, 012005. 
(a)
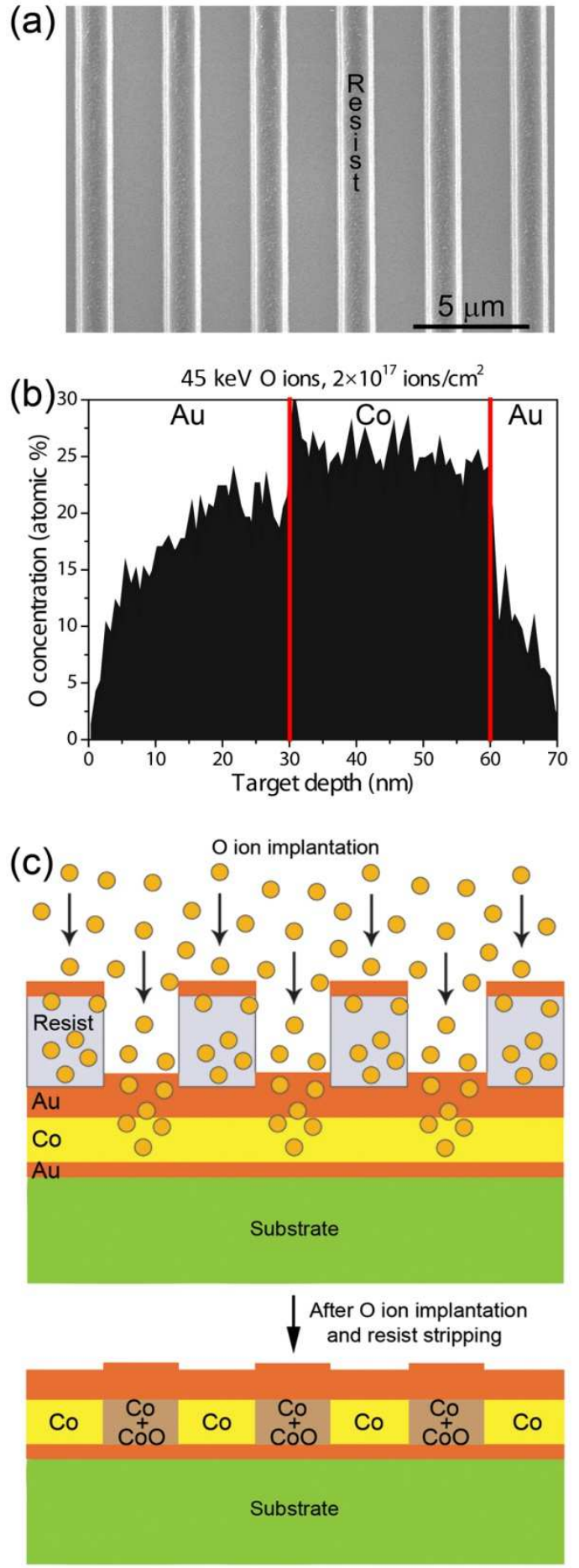

Figure 1. (a) Scanning electron microscopy image, taken with secondary electrons, of the lithographed resist mask. (b) $\mathrm{O}$ depth profile along sample depth of the unprotected areas, simulated by TRIM, ${ }^{[32]}$ for $45 \mathrm{keV} O$ ions and a fluence of $2 \times 10^{17}$ ions $/ \mathrm{cm}^{2}$. (c) Cartoon showing the implantation process and the cross section view of the laterally modulated microscale lines in which $\mathrm{Co}$ and $\mathrm{Co}-\mathrm{CoO}$ areas are alternating. 


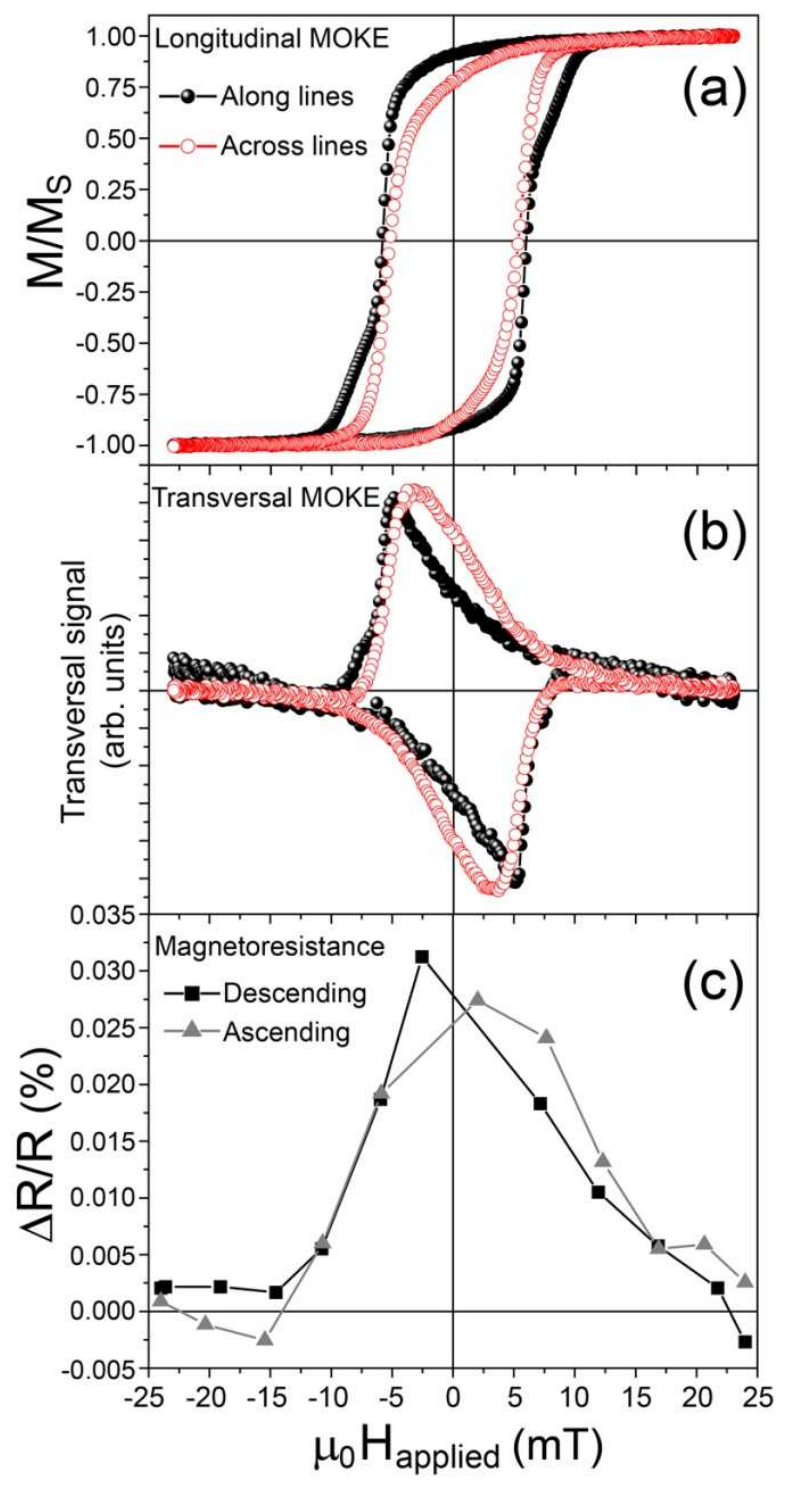

Figure 2. Room temperature: (a) and (b) are the longitudinal and non-normalized transversal magnetooptic Kerr effect (MOKE) measurements, respectively, along and across the lines. (c) is the magnetoresistance measurement with the current and magnetic field applied along the lines. 




(b) $3 \mathrm{mT}$

(c) $4.5 \mathrm{mT}$
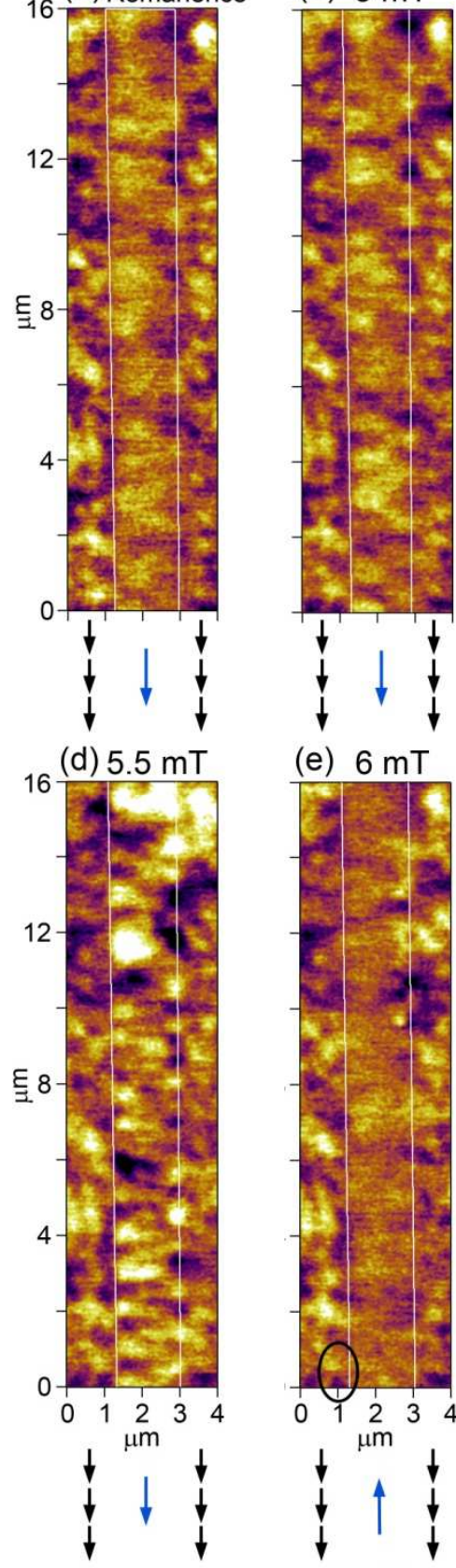

(e) $6 \mathrm{mT}$
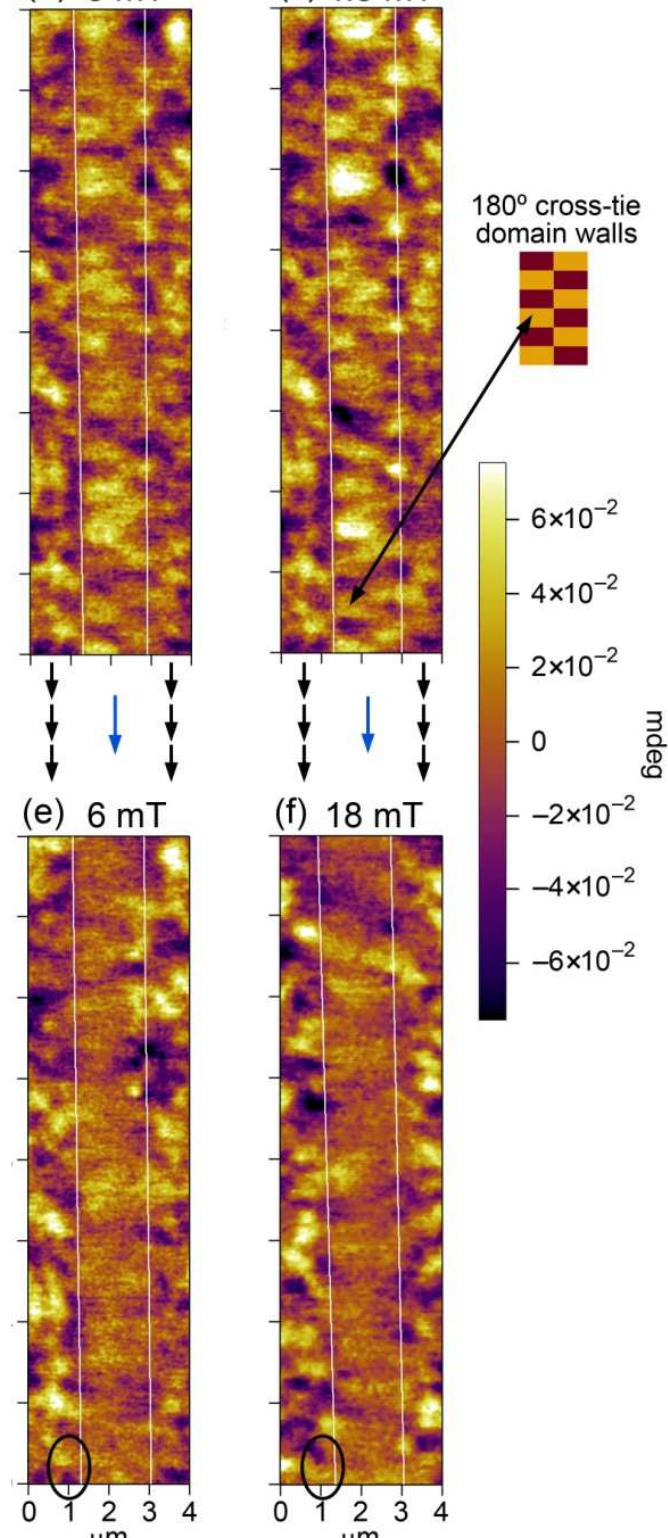

응 


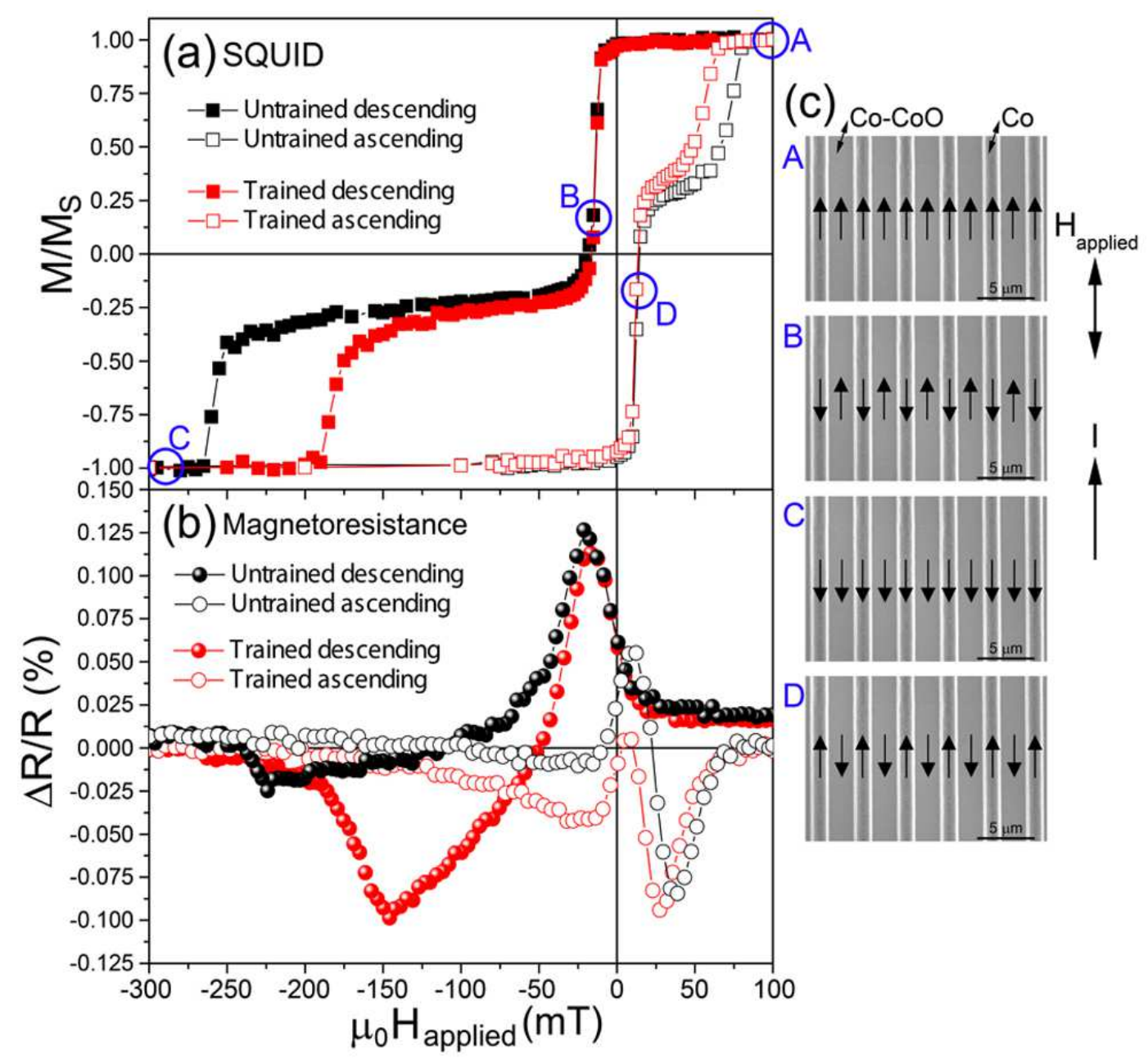

Figure 4. (a) Untrained and trained superconducting quantum interference device (SQUID) measurements along the lines. (b) Untrained and trained magnetoresistance measurements with the current and applied magnetic field along the lines and (c) cartoon showing the magnetization configuration at dedicated applied magnetic fields. B and D represent stripe domains with $180^{\circ}$ Néel domain walls at the interfaces. 

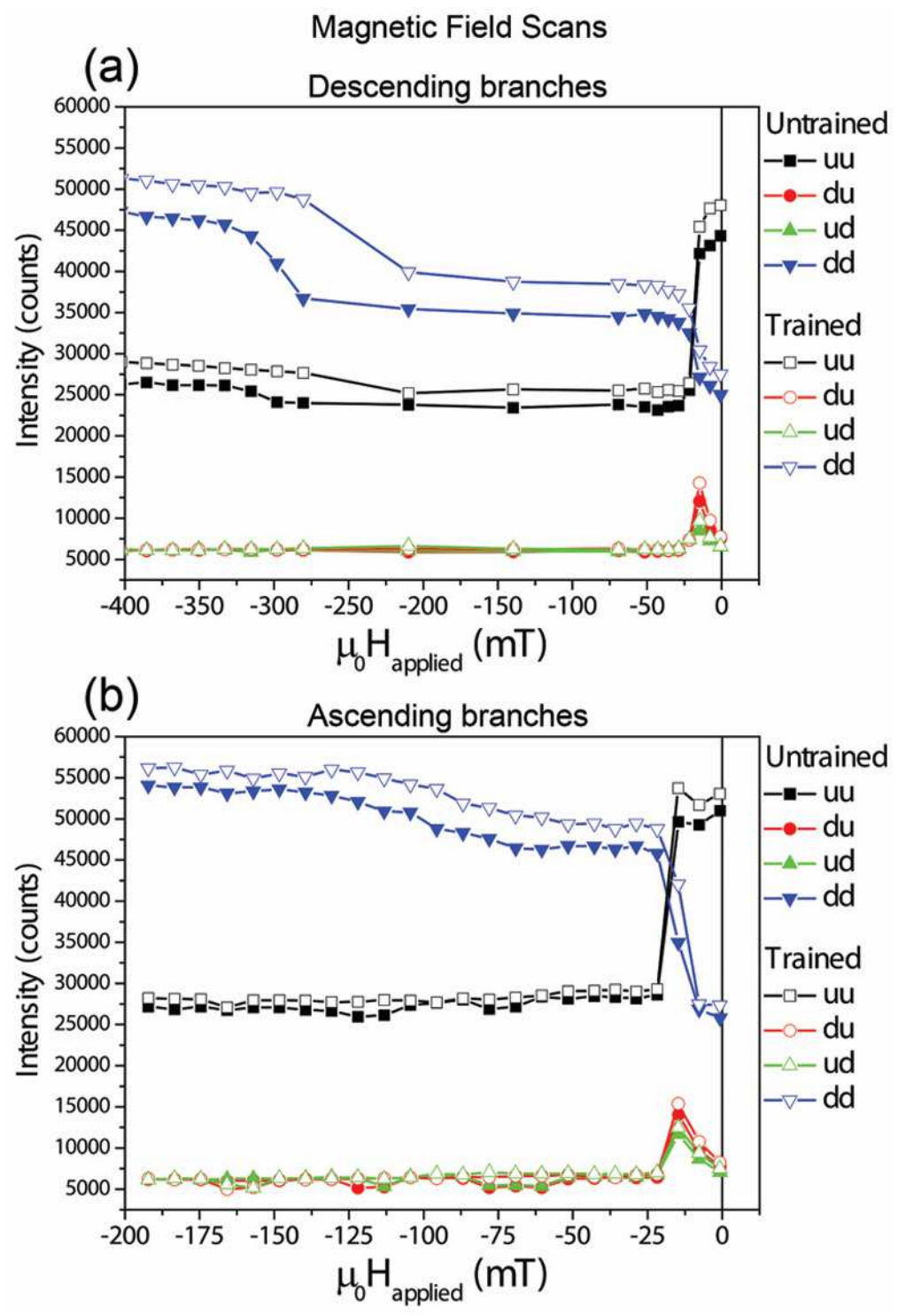

Figure 5. (a) and (b) $10 \mathrm{~K}$ polarized neutron reflectometry magnetic field scans along the lines of the untrained and trained descending and ascending branches, respectively. These measurements show both the raw specular and off-specular signal. 
(a) uu

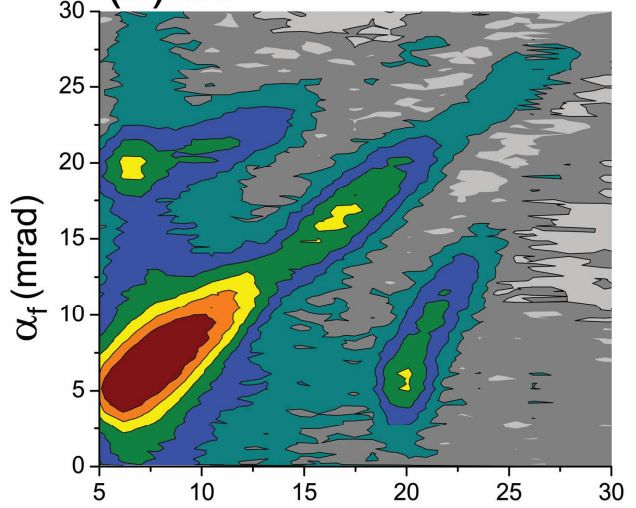

(c) ud $\alpha_{i}(\mathrm{mrad})$

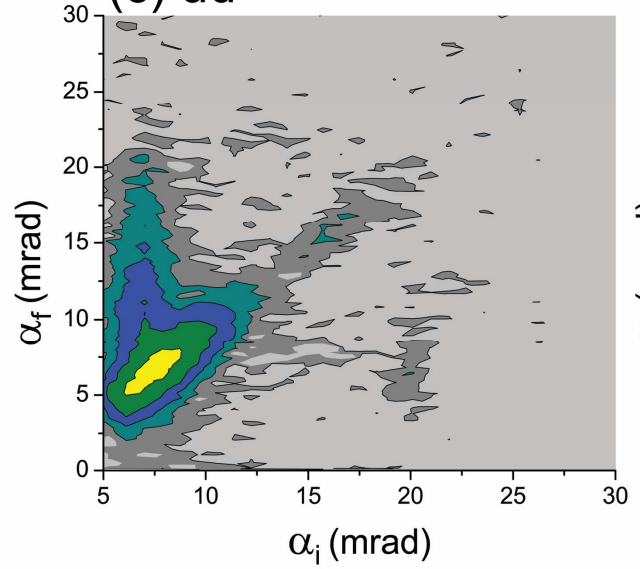

(b) dd

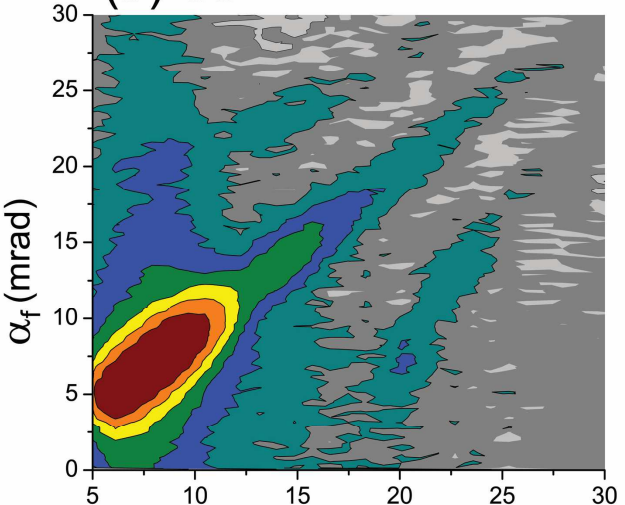

(d) du $\alpha_{i}$ (mrad)

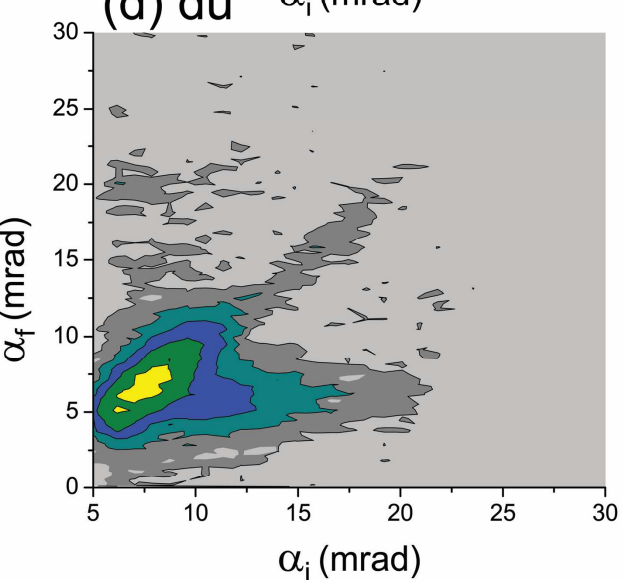

$-0.6 \mathrm{mT}$

$10 \mathrm{~K}$

$\log$ (Intensity)

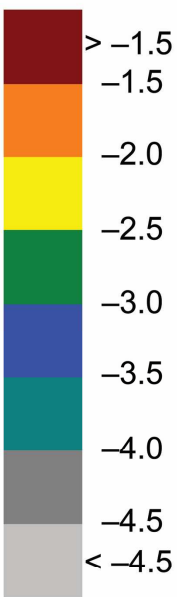

Figure 6. Polarized neutron reflectometry raw $\alpha_{i}-\alpha_{f}$ maps corresponding to uu (a), dd (b), ud (c) and ud (d) neutrons, taken at $10 \mathrm{~K}$ in a magnetic field of $-0.6 \mathrm{mT}$ applied along the lines. 


\section{Table of Contents}

Ion implantation through lithographed masks is shown to be an advantageous method to prepare laterally, compositionally and magnetically, modulated samples with tunable giant magnetoresistance and potential uses for spintronic research and applications. A lateral pattern consisting of alternating $\mathrm{Co}(1.6 \mu \mathrm{m}$-wide) and $\mathrm{Co}-\mathrm{CoO}(2.4 \mu \mathrm{m}$-wide) lines has been prepared by oxygen ion implantation into a pre-lithographed Au-sandwiched Co thin film.

Lateral magnetically modulated multilayers

E. Menéndez*, H. Modarresi, C. Petermann, J. Nogués*, N. Domingo. H. Liu, B. J. Kirby, B. Maranville, J. A. Borchers, A. S. Mohd, Z. Salhi, E. Babcock, S. Mattauch, L. Lagae, C. Van Haesendonck, A. Vantomme, K. Temst*

Lateral magnetically modulated multilayers by combining ion implantation and lithography

ToC figure

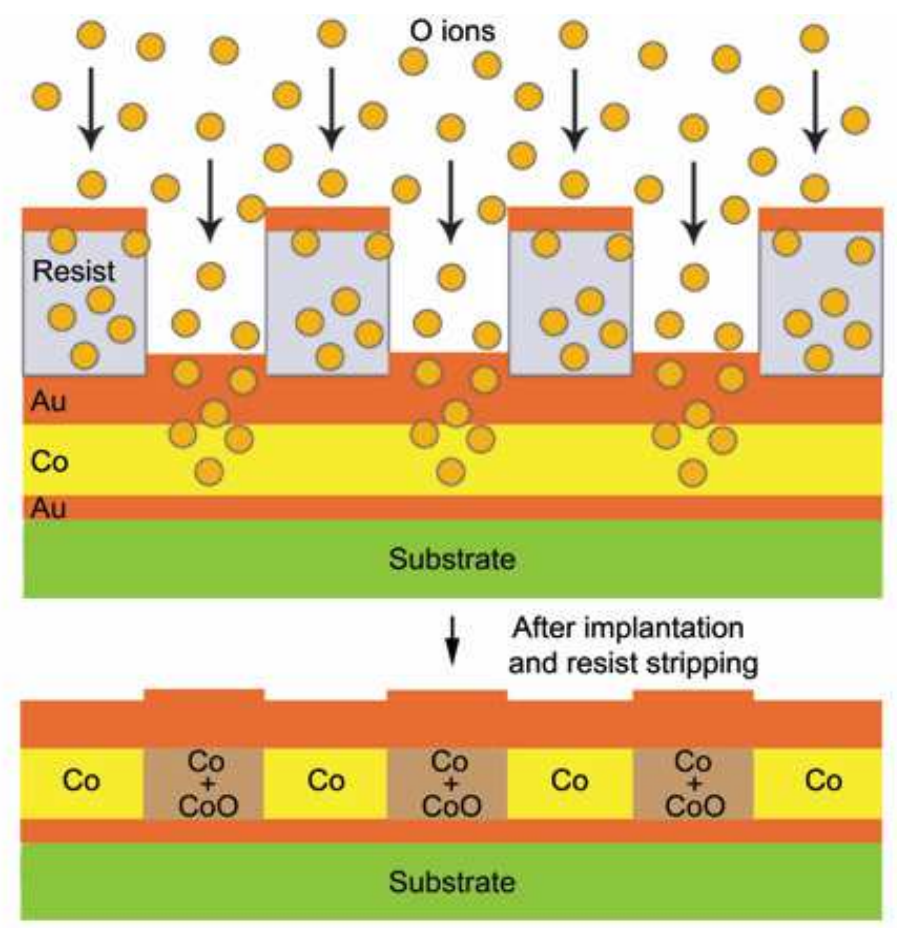

